\section{Utility of the low-dose short Synacthen test in diagnosis of adrenal insufficiency in outpatients with nonspecific symptoms}

\author{
Anne Corbould,1,2 Matthew Jarvis,3 \\ Joanne Campbell, 1 Deborah Kunde, ${ }^{4}$ \\ Wade Clarkson, ${ }^{4}$ Denise Burns ${ }^{4}$ \\ 1Department of Medicine, \\ Launceston General Hospital, Tasmania; \\ 2Faculty of Health Science, University of \\ Tasmania; ${ }^{3}$ Royal Adelaide Hospital, SA; \\ 4Department of Pathology, Launceston \\ General Hospital, Tasmania, Australia
}

\section{Abstract}

Short Synacthen tests are used to diagnose primary and secondary adrenal insufficiency. The standard test uses supraphysiological adrenocorticotropic hormone $(250 \mu \mathrm{g})$. The low-dose test $(1 \mu \mathrm{g})$ was proposed to be more sensitive. In outpatients with nonspecific symptoms referred for exclusion of adrenal insufficiency, we assessed whether the lowdose test was an appropriate initial investigation. Results of 233 low-dose tests were obtained from pathology database 2007-2009. Clinical data were available for 169 patients: 37 taking glucocorticoids or with known pituitary/adrenal disease were excluded. Among the remaining 132 patients, $26 \%$ failed using stimulated cortisol $\geq 500 \mathrm{nmol} / \mathrm{L}$ and $45 \%$ failed at $\geq 550 \mathrm{nmol} / \mathrm{L}$. These rates of apparent adrenal insufficiency are $\sim 50$ fold above expected. In conclusion, the low-dose test, using conventional criteria for normality, is abnormal in a large proportion of patients with nonspecific symptoms. Until criteria for normal cortisol response to low-dose Synacthen are clarified, alternative tests of adrenal function are advised in this clinical setting.

\section{Introduction}

The short Synacthen [tetracosactrin i.e. synthetic adrenocorticotropic hormone (ACTH) 1-24] test is routinely used to diagnose adrenal insufficiency, both primary and secondary (i.e. due to hypothalamic-pituitary disorders). Use of the short Synacthen test in assessment of secondary adrenal insufficiency relies on adrenal gland atrophy due to impaired endogenous ACTH secretion and thus cannot be used acutely after hypothalamic-pituitary injury.
The standard-dose test (250 $\mu \mathrm{g}$ Synacthen) uses a supraphysiological ACTH dose. The lowdose test (1 $\mu$ g Synacthen) was proposed as a more sensitive test: its use has been advocated in diagnosis of secondary adrenal insufficiency due to chronic hypothalamic-pituitary disease and glucocorticoid administration. ${ }^{1}$ However, technical issues such as requirement for dilution of Synacthen prior to administration and potential binding of ACTH onto plastic injection devices, ${ }^{2}$ as well as lack of consensus on the normal cortisol response to 1 $\mu \mathrm{g}$ Synacthen, ${ }^{3-5}$ have prevented universal acceptance of this test. A stimulated cortisol cutoff value of $500-550 \mathrm{nmol} / \mathrm{L}$ is conventionally used to indicate a normal response in the low-dose Synacthen test, however a recent study in healthy adults reported the lowest cortisol response to $1 \mu \mathrm{g}$ Synacthen was 345 $\mathrm{nmol} / \mathrm{L} .{ }^{5}$

We had the opportunity to evaluate the lowdose Synacthen test in a unique population i.e. outpatients presenting with nonspecific symptoms, no known adrenal/pituitary disease, and not taking glucocorticoids. Until 2009, the default Synacthen test offered by our regional pathology laboratory (serving a population of $\sim 100,000$ ) was low-dose: thereafter, the standard-dose Synacthen test became the default test. We aimed to investigate the frequency that the low-dose test was abnormal in patients referred for investigation of nonspecific symptoms in the period May 2007 - April 2009 in order to determine whether this was an appropriate initial test in this clinical setting. Confirmation of adrenal insufficiency with additional tests (standard-dose Synacthen test or insulin tolerance test), or investigation of underlying diagnoses in those patients with an abnormal low-dose Synacthen test, was beyond the scope of this study. We also investigated if measurement of basal serum cortisol alone would have been sufficient for diagnosis in a significant number of patients, thus avoiding the need for a dynamic test.

\section{Materials and Methods}

The results of the Synacthen tests and clinical information on the request forms were obtained from the Pathology Department database at Launceston General Hospital for the period May 2007 - April 2009. All data obtained were non-identifiable. In accordance with the National Statement on Ethical Conduct in Human Research, Human Ethics Committee approval was not required.

For the test, $250 \mu \mathrm{g}$ Synacthen (Novartis Pharmaceuticals, Switzerland) was diluted in $50 \mathrm{~mL}$ normal saline, stored at $4^{\circ} \mathrm{C}$ in glass for $\leq 1$ month, then further diluted to $1 \mu \mathrm{g} / \mathrm{mL}$ in a disposable polyethylene syringe on the morn-
Correspondence: Dr Anne Corbould, Department of Medicine, Launceston General Hospital, Charles Street, Launceston, Tasmania 7250, Australia.

Tel. +61.363487111 - Fax +61.363362433.

E-mail: acorbould@yahoo.com

Key words: adrenal insufficiency/diagnosis, cosyntropin/diagnostic use, humans, outpatients.

Contributions: AC, JC, WC and DB contributed to the intellectual planning of the project. $\mathrm{AC}, \mathrm{MJ}$ and DK contributed to preparation of the data base and analysis and interpretation of data. Initial preparation of the manuscript was carried out by $\mathrm{AC}$ with revisions and final approval from all other authors.

Conflict of interests: the authors report no conflict of interests.

Received for publication: 20 September 2012. Revision received: 27 October 2012.

Accepted for publication: 27 October 2012.

This work is licensed under a Creative Commons Attribution NonCommercial 3.0 License (CC BYNC 3.0)

(C) Copyright A. Corbould et al., 2012

Licensee PAGEPress, Italy

Endocrinology Studies 2012; 2:e5

doi:10.4081/es.2012.e5

ing of the test. One $\mu g$ of Synacthen was administered i.v. via a butterfly infusion set at 0900 - 0930 hrs with measurement of serum cortisol immediately pre-dose and 30 minutes post-dose. The 30 minute post-Synacthen cortisol value was used for interpretation of the test as this is a common protocol used in published studies,,$^{3,4}$ and there is some evidence that the 30 minute cortisol may be superior to the 20 minute value. ${ }^{4}$ Moreover, $84 \%$ of peak responses occur at 30 minutes after administration of low dose Synacthen. ${ }^{5}$ Serum cortisol was assayed using an automated competitive immunoassay (VITROS ECi, Ortho-Clinical Diagnostics, Raritan, NJ, USA). The limit of quantitation of this assay is $\sim 5 \mathrm{nmol} / \mathrm{L}$, and intra- and inter-assay coefficient of variation $2.2 \%$ and $5.1 \%$, respectively.

We analysed stimulated cortisol cutoff values of $500 \mathrm{nmol} / \mathrm{L}$ and $550 \mathrm{nmol} / \mathrm{L}$, as well as the newly proposed cutoff of $345 \mathrm{nmol} / \mathrm{L} .^{5}$ The increment of serum cortisol after Synacthen was not considered as a criterion. Regarding basal cortisol levels, an early morning serum cortisol $<100 \mathrm{nmol} / \mathrm{L}$ was considered to be diagnostic of adrenal insufficiency and a cortisol $\geq 450 \mathrm{nmol} / \mathrm{L}$ to indicate normal hypothalamic-pituitary-adrenal axis function. ${ }^{6}$ 


\section{Results}

A total of 233 patients had low-dose Synacthen tests performed during the 2 year period. All but 3 were on outpatients i.e. referred from primary care $(n=87,37 \%)$, outpatient clinics or physician's consulting rooms. Clinical data were recorded on the pathology request form for 169 patients and no clinical data was recorded for 64 . Where clinical information was available, patients currently or recently on glucocorticoids or with known pituitary/adrenal disease were excluded from the analysis $(n=37)$. No patients were stated to be taking estrogen therapy. Among the remaining 132 patients in whom clinical information was available, the most common reasons given for the test (accounting for $75 \%$ of test requests) were fatigue/lethargy $(n=42$, i.e. $32 \%$ of patients), hypotension/syncope $(n=19,14 \%)$, possible hypoadrenalism $(n=18,13 \%)$, unexplained vomiting/abdominal pain $(n=8,6 \%)$, unexplained weight loss $(n=5,6 \%)$ and hyponatraemia $(n=5,4 \%)$. In the remaining 35 patients, a variety of other indications were stated, including previous abnormal cortisol levels, salt craving, type 1 diabetes.

The results are summarized in Table 1 . Among patients with clinical information, (i.e. nonspecific symptoms, not taking glucocorticoids and without known adrenal or pituitary disease), $45 \%$ failed to achieve stimulated serum cortisol $\geq 550 \mathrm{nmol} / \mathrm{L}$ and $26 \%$ failed to achieve stimulated cortisol $\geq 500 \mathrm{nmol} / \mathrm{L}$. At a cortisol cutoff of $345 \mathrm{nmol} / \mathrm{L}, 8 \%$ failed to achieve an adequate response. In the group without clinical information, the results were similar (data not shown). Basal serum cortisol levels were considered diagnostic in 25 patients (i.e. 19\%) in whom clinical information was available: all of the patients in this group with basal cortisol $<100 \mathrm{nmol} / \mathrm{L}(n=5)$ failed to achieve a stimulated cortisol $\geq 500$ $\mathrm{nmol} / \mathrm{L}$ (range $50-415 \mathrm{nmol} / \mathrm{L}$ ). The indications given for testing in these patients were hypotension, fatigue, probable Addison's Disease, previously low cortisol, and diabetes. The basal cortisol was $\geq 450 \mathrm{nmol} / \mathrm{L}$ in 20 patients in this group, all of whom achieved stimulated cortisol $\geq 500 \mathrm{nmol} / \mathrm{L}$ (range 540 $1260 \mathrm{nmol} / \mathrm{L})$.

\section{Discussion}

Symptoms of both primary and secondary adrenal insufficiency may be nonspecific but diagnosis is crucial as these disorders can be fatal if untreated. In the investigation of primary adrenal insufficiency, the standard-dose Synacthen test is conventionally used: the lowdose test has been advocated by some for the diagnosis of partial or subtle primary adrenal insufficiency, however data regarding the performance of the test for this clinical indication are lacking. 1 The performance of both the standard-dose and low-dose Synacthen tests has been extensively studied in patients with hypothalamic-pituitary disorders but the relative utility of the tests is still debated.1,3,4 The minimum stimulated serum cortisol level that defines a normal response to Synacthen varies widely in the literature.1,3,5 There is also evidence of significant variation between different cortisol assays. ${ }^{7}$

We evaluated the low-dose Synacthen test in outpatients presenting with nonspecific symptoms such as fatigue and lethargy, no known adrenal or pituitary disease and not taking glucocorticoids. We are not aware of any similar data in the published literature. In our study, the low-dose Synacthen test was abnormal in $\sim 26 \%$ or $\sim 45 \%$ of patients using stimulated cortisol cutoff values of $500 \mathrm{nmol} / \mathrm{L}$ and 550 $\mathrm{nmol} / \mathrm{L}$, respectively. These data indicate that using conventional stimulated cortisol cutoff values, the frequency of newly diagnosed primary or secondary adrenal insufficiency is 17 30 cases per year in our population of 100,000. This contrasts with the published incidence of primary adrenal insufficiency of 4.7-6.2 per million per year. ${ }^{8}$ If our cases represented secondary adrenal insufficiency, the most common cause of which is administration of glucocorticoids, the number of new cases each year would exceed the published prevalence of secondary adrenal insufficiency i.e. 150-280 per million. ${ }^{8}$ Using the criterion of stimulated cortisol cutoff of $345 \mathrm{nmol} / \mathrm{L}$ proposed by a recent study, ${ }^{5}$ adrenal insufficiency would be diagnosed in $8 \%$ i.e. 5 new cases per year, which is still 10 fold higher than the expected incidence of primary adrenal insufficiency. However, confirmation by additional investigators of the utility of the cortisol cutoff of $345 \mathrm{nmol} / /$ for the low-dose Synacthen test is necessary before this can be universally adopted.

We also investigated whether an early morning serum cortisol level could reduce the need for dynamic testing of adrenal function in out- patients i.e. serum cortisol $<100 \mathrm{nmol} / \mathrm{L}$ or $\geq 450 \mathrm{nmol} / \mathrm{L}$. Our data showed that dynamic testing of adrenal function could have been avoided in less than $20 \%$ of outpatients, similar to that shown in a study by le Roux et al. ${ }^{9}$

A limitation of our study is that we had access to limited clinical details. We excluded patients stated to be currently or recently on glucocorticoids. If such patients had been included in the analyses due to incomplete information, this would have the effect of increasing the number of patients who failed the low-dose Synacthen test. However this would be an unlikely explanation for the frequency of adrenal insufficiency as diagnosed on low-dose Synacthen test in our population i.e. up to 50 fold above expected rates. Another limitation of our study is that we did not have information on subsequent investigation of the patients i.e. to confirm or refute a diagnosis of adrenal insufficiency in those with an abnormal low-dose Synacthen test. However, the frequency of abnormal test results in our study population argues strongly in favour of frequent false positive results: the proposition that the true incidence of adrenal insufficiency in outpatients with nonspecific symptoms is up to $45 \%$ is untenable. Gender, age and body mass index were not available in our patients, although a recent study has shown that these factors did not affect the cortisol response to Synacthen. ${ }^{5}$

\section{Conclusions}

We conclude that the probability of the lowdose Synacthen test being abnormal in outpatients with nonspecific symptoms is $26-45 \%$, using conventional criteria for a normal cortisol response i.e. $500 \mathrm{nmol} / \mathrm{L}$ or $550 \mathrm{nmol} / \mathrm{L}$, respectively. It is important that clinicians are aware of this finding, given that for most patients, a second round of investigations will then be required to confirm adrenal insufficiency (e.g. standard-dose Synacthen test or insulin tolerance test), thus causing additional patient

Table 1. Low-dose Synacthen test in patients with clinical data available.

\begin{tabular}{lc} 
& $\begin{array}{c}\text { Serum cortisol } \mathrm{nmol} / \mathrm{L} \\
\mathrm{n}=132\end{array}$ \\
baseline (mean \pm standard deviation) & $312 \pm 173$ \\
$+30 \mathrm{~min}$ (mean \pm standard deviation) & $583 \pm 182$ \\
\hline$<345$ at $+30 \mathrm{~min}(n)$ & $10(8 \%)$ \\
$<500$ at $+30 \mathrm{~min}(n)$ & $35(26 \%)$ \\
$<550$ at $+30 \mathrm{~min}(n)$ & $59(45 \%)$ \\
$<100$ at baseline $(n)$ & $5(4 \%)^{*}$ \\
$\geq 450$ at baseline $(n)$ & $20(15 \%)^{* *}$ \\
\hline 'Stimulated cortisol $<500 \mathrm{nmol} / \mathrm{L}$ (range $50-415 \mathrm{nmol} / \mathrm{L})$. "Stimulated cortisol $\geq 500 \mathrm{nmol} / \mathrm{L}($ (range $540-1260 \mathrm{nmol} / \mathrm{L})$.
\end{tabular}


inconvenience and laboratory expense. Until the criteria for a normal cortisol response to low-dose Synacthen have been re-evaluated, it would seem prudent to choose an alternative test of adrenal function for the initial investigation of patients with nonspecific symptoms.

\section{References}

1. Dickstein G, Saiegh L. Low-dose and highdose adrenocorticotropin testing: indications and shortcomings. Curr Opin Endocrinol Diab Obes 2008;15:244-9.

2. Murphy H, Livesey J, Espiner EA, Donald RA. The low dose ACTH test- a further word of caution. J Clin Endocrinol Metab 1998;83:712-3.

3. Dorin RI, Qualls CR, Crapo LM. Diagnosis of adrenal insufficiency. Ann Intern Med 2003;139:194-204.

4. Kazlauskaite R, Evans AT, Villabona CV, et al. Corticotropin tests for hypothalamicpituitary-adrenal insufficiency: a metaanalysis. J Clin Endocrinol Metab 2008;93:4245-53.

5. Karaca Z, Lale A, Tanriverdi F, et al. The comparison of low and standard dose ACTH and glucagon stimulation tests in the evaluation of hypothalamo-pituitaryadrenal axis in healthy adults. Pituitary 2011;14:134-40.

6. Inder WJ, Hunt PJ. Gluocorticoid replace- ment in pituitary surgery: guidelines for perioperative assessement and management. J Clin Endocrinol Metab 2002;87: 2745-50.

7. Clark PM, Neylon I, Raggatt PR, et al. Defining the normal cortisol response to the short Synacthen test: implications for the investigation of hypothalamic-pituitary disorders. Clin Endocrinol 1998;49: 287-92.

8. Arlt W, Allolio B. Adrenal insufficiency. Lancet 2003;361:1881-93.

9. Le Roux CW, Meeran K, Alaghband-Zadeh J. Is a 0900-h serum cortisol useful prior to a short Synacthen test in outpatient assessment? Ann Clin Biochem 2002;39: 148-50. 